\title{
Statistical Profiling of Site Wind Resource Speed and Directional Characteristics
}

\author{
Bruce Stephen, Stuart Galloway, David McMillan, Lucy Anderson and Graham Ault
}

Abstract- Construction of a wind farm without a reliable plant margin forecast can jeopardize potential returns on investment from the outset. Meteorological and topological factors influence the wind characteristics across any site which in turn affects wind farm output, critical for localized generation, and also the dynamic loading of the turbine structure. The models developed in this paper follow the generally advocated use of probability density estimation as a means of representing wind resource characteristics but, owing to differences, in characterization that may be encountered, do not assume a single distribution form across all sites. A mixture modelling approach is adopted that removes the need for choosing distribution forms on a site by site basis. Advancing previous work constructing statistical distributions over congruent wind speed and direction observations of the wind resource characteristics at a given site, the proposed model, as a consequence of using a mixture distribution, captures both recurring regimes in the site behaviour along with their frequency of occurrence. Preliminary results using data sets from a diverse range of locations in Scotland demonstrate the variation in the forms of model learned; comparisons of the model with current and alternate practices are given through visualization and resource assessment illustrations.

Index Terms - Wind speed estimation; Wind direction estimation; Extreme event recognition

\section{Introduction}

Meteorological conditions are likely to be the most complex and crucial factor influencing choice of wind farm location with optimal placement being an important business decision, one of maximizing generation output and minimizing potential costs thus reducing the time to recoup the initial investment. Ideally, planners would want to be able to incorporate maximal knowledge gleaned from existing sites to anticipate operational issues and model these to find optimal estimates of resource characteristics and foresee operating issues that the surrounding environment may pose to assist in this decision making process. In line with the EU Renewable Energy Directive, the UK must endeavour to generate $15 \%$ of its total energy from renewable sources by 2020 , a target which a future UK energy system may meet with a highly decentralized network [1]. Through the widespread use of Distributed Energy Resources (DERs), support to the current electricity and gas networks in times of peak load would assist in delivering the stable energy network that is required, whilst meeting EU targets. Segregating the energy system into a number of constituent elements of smaller 'local' decentralized energy networks, where each portion has its own load, generation and network considerations $[2,3,4]$, greatly reduces the control problem surrounding DER integration found in current top-down network topologies. This ultimately results in the need for accurate weather profiling and forecasting so that the benefits that distributed generation (DG) such as wind turbines (both micro and large scale plant) can be fully exploited by identifying the less than subtle differences in demand across areas with different weather profiles. It has been shown that better characterisation of the complexities of the wind regimes results in superior forecasting performance [5]. A further important application for understanding the complexity of a site wind regime is the possible impact of the turbine wind loading on the failure rates of pitch and bearing systems $[6,7]$. Producing a statistical model of the meteorological characteristics of a site captures these traits and the variance or noise associated with them through probability density estimates allowing higher level analyses such as model comparison to find sites similar to optimally placed ones, or to track longer period site characteristics; and utilizing the model for simulation of future generation output from the site. A number of different probability distributions have been used to model wind speeds both for wind energy and general meteorological purposes. Key criteria for selection of a suitable 
distribution include representation of a continuous random variable, the random variable must be strictly positive and there must be a means of accommodating the occurrence of zeros - also known as null wind speeds. Although many parametric distributions fit these criteria, estimation of the parameters of a candidate distribution must also be computationally tractable.

A traditional choice for the characterization of wind speeds has been the 2-parameter Weibull distribution [8, 9]; however, it is unable to properly represent regimes with a large occurrence of null wind speeds and, as a single mode distribution, bimodality. This conventional assumption of unimodality for modelling wind speed can distort the true power estimate of a wind resource as well as obscure some of the potentially useful statistical features of it. For example, a site featuring steady low speed wind could be skewed by the infrequent occurrence of winds of a higher speed. In a single mode distribution, such as the Weibull, this situation would result in the expected wind speed being shifted upwards thus overestimating the potential generation capacity of the site. Also, the potentially unusable high winds, which would necessitate the feathering of turbine blades, would be hidden. Such a scenario would result in generation capacity being overestimated. A concrete example of how wind speeds can exhibit multiple behaviours according to climatic or topographic features is provided in [10]. In this study of multimodal wind speed characteristics resulting from two different topographically influenced meteorological regimes, two Weibull distributions were used to characterize the site. The clear bimodality observed and modelled in this study highlighted how inappropriate a single mode distribution could be. In a comprehensive comparison, [11] reviewed wind energy case studies in the Canary Islands where the combination of trade winds and sea breezes across the islands has created both unimodal and bimodal wind speed distributions. Many approaches have assumed that there will only be two modes and/or tangents to the wind speed distribution when in reality there is no way of automatically knowing this will be the case; [9] demonstrated the variability of offshore wind characteristics and found that different distribution models perform well in different circumstances which makes it essential that there be models that are as adaptable as possible to the true form of the wind speed probability density function (PDF).

In addition to wind speed estimates, it is important for any wind model to properly reflect directional observations as these too can exhibit multimodality in terms of the prevailing direction $[12,13]$. Directional variability is used as an indicator of turbulence in Pasquill classes [14] and overly turbulent flow can affect the consistency of generation at a site irrespective of wind speed [15]. Additionally, structural loading is influenced by turbulent flow [16] which can dictate the operational bounds of a turbine and shorten its service life [17].The current standard pertaining to wind turbine performance, IEC 61400-12 [18], does not elaborate on directional or modal characteristics of wind direction. In [18] the use of a unimodal Weibull distribution is advocated to model the wind speed at a given site, recommending a bin size (wind speed magnitude) of $0.5 \mathrm{~m} / \mathrm{s}$ but gives no such guidance for directional characteristics. Directional information was touched on in [19] with reference to [18]. The popular graphical representation of the occurrence of particular speed/direction values, the wind rose is equivalent to a circular histogram, although there is no way of quantifying the differences or similarity between sites. While the wind rose captures the directional aspects to an extent, it is through a binned representation that observes the general prevalent wind direction.

Updating earlier works on directional modelling, [20] used a mixture of von Mises distributions to capture multi-modality in wind direction in various sites in the Canary Islands. Through goodness of fit criteria, [20] demonstrated that comparison of the von Mises Mixture model with the bivariate Gaussian model showed that von Mises proved to be the superior fit. More recently, [21] proposed a new circular probability distribution based on the generalization of von Mises that brought speed and direction into a single model. It showed this distribution to be a better fit than the mixture of von Mises as used in $[20,22]$. Neither model accommodates the situation where there can be no directional measurement associated with a null wind speed and in both cases, speed and direction of wind are assumed to independent. Assumptions of independence between wind speed and direction are commonplace $[21,22]$ and there are good reasons for this. Where no realistic model of 
dependence can be assumed it is better to cover all eventualities through the distribution choice by assuming independence at the expense of blanket coverage of outlier combinations. Assuming a joint mixture distribution however allows both a piecewise fitting of both the density function as well as its dependence between variables.

In this paper, a model is presented which builds on the independent joint distributions models of [21] and [22]. Since there are no heuristics or rules of thumb for how a site will be characterized, with additional uncertainty introduced by offshore sites, any model used must be flexible enough to adapt to all eventualities through automated model selection and parameter estimation from observed data. Model generalisation is permitted through the aggregation of exemplar data used for model training. Mixtures of cylindrical probability distributions are used to capture particular regimes present in the wind resource at a given site; the complexity of the model, implied by the number of regimes at a given site, is controlled by a computationally viable realization of Bayesian model selection. Like [18] a formulation of the algorithm for learning the parameters of these models from data is presented. An example of how this approach would be used for site resource assessments is provided along with the benefits and ways in which these models can be employed which are discussed in the concluding remarks.

\section{Sites Used}

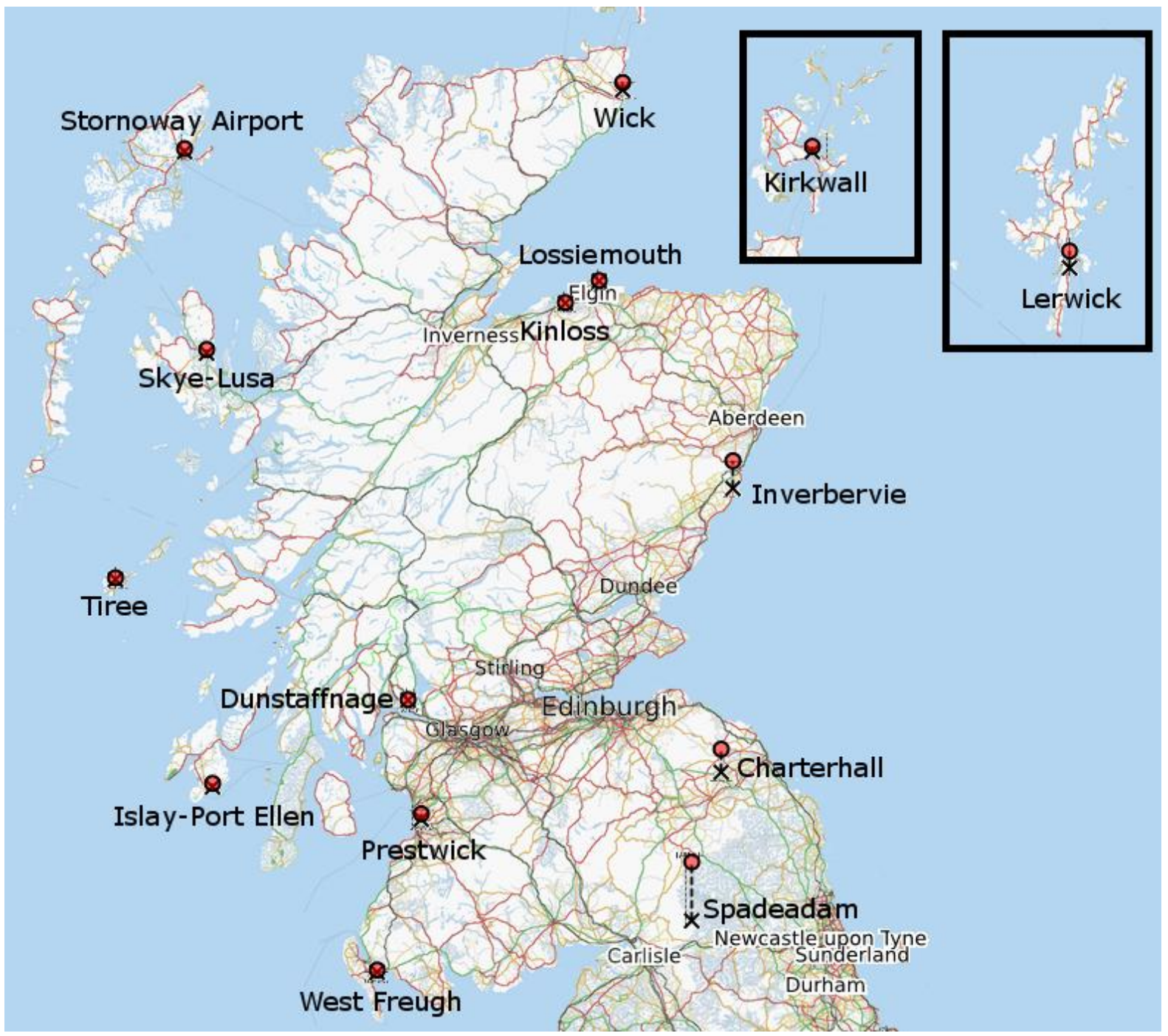

Figure 1: Geographic locations of the sites used in this paper. The stem lengths indicate higher altitude stations. 
In [10] situations were demonstrated where windspeeds at La Ventosa could be multimodal and hence required specialized distributions; [23] showed for a number of offshore sites in the Eastern Mediterranean where the distribution of windspeeds took an arbitrary shape; in the study of the Canarian Archipelago, [11] showed how some distributions had a more pronounced mode than any parametric distribution available and in Gran Canaria in particular, bimodality. Lastly [6] demonstrated how, for a number of offshore sites, the form of the distribution varied widely and could not be assumed in advance of analysis. In [11], [24] and [10] the influence of topography and location of a site has on wind resource characteristics is noted. For this reason, a selection of sites is used in this work. A set of hourly averaged meteorological observations over 4 years (2003-2006) were taken from a diverse selection of weather stations positioned across Scotland. As shown in table 1 and figure 1, the stations were chosen to capture the different topological and climatic characteristics of their locations; consequently islands, high ground and both coasts are featured.

\begin{tabular}{|c|c|}
\hline Station & Location/Characteristics \\
\hline Charterhall & Inland \\
\hline Dunstaffnage & Highland, Coastal \\
\hline Islay & Island, Atlantic \\
\hline Kirkwall & Island, North Sea \\
\hline Kinloss & Highland, Coastal, North Sea \\
\hline Inverbervie & Coastal, North Sea \\
\hline Lerwick & Island, North Sea \\
\hline Lossiemouth & Highland, Coastal, North Sea \\
\hline Prestwick & Lowland, Coastal, Irish Sea \\
\hline Spadeadam & Inland, High Ground \\
\hline Stornoway & Island, Atlantic \\
\hline Tiree & Island, Atlantic \\
\hline West Freugh & Lowland, Coast, Irish Sea \\
\hline Wick & Highland, Coastal, North Sea \\
\hline
\end{tabular}

Table I: Stations Used

Two exemplary sites are represented as wind directional and speed histograms in figure 2: Charterhall and Tiree. The groupings in these examples demonstrate that there is more than one directional regime in action at these sites in a single month as well as more than one regime for windspeed at one of the sites. Charterhall has a variable but dominant south-westerly $\left(\sim 240^{\circ}\right)$ wind direction with a less frequent northern occurrence $\left(\sim 10^{\circ}\right)$. Three possible modes can be identified from the windspeed histogram at 8,15 and 30 knots. Tiree has an even more variable westerly direction and also a northern mode; windspeed at Tiree is bi-modal with a void at 20 knots. Although there is valuable information to be obtained from histograms, the dependency between the windspeed and direction regimes is not represented. To illustrate the importance of these, figure 3 shows 2-parameter Weibull distributions fitted to the 12 directional sectors at Charterhall. Most of the distributions are distinctly parameterized implying that a single windspeed distribution cannot 
be reliably assumed for a given site. While these are individual examples, it does reflect the need to have a single model that can adapt to all eventualities.
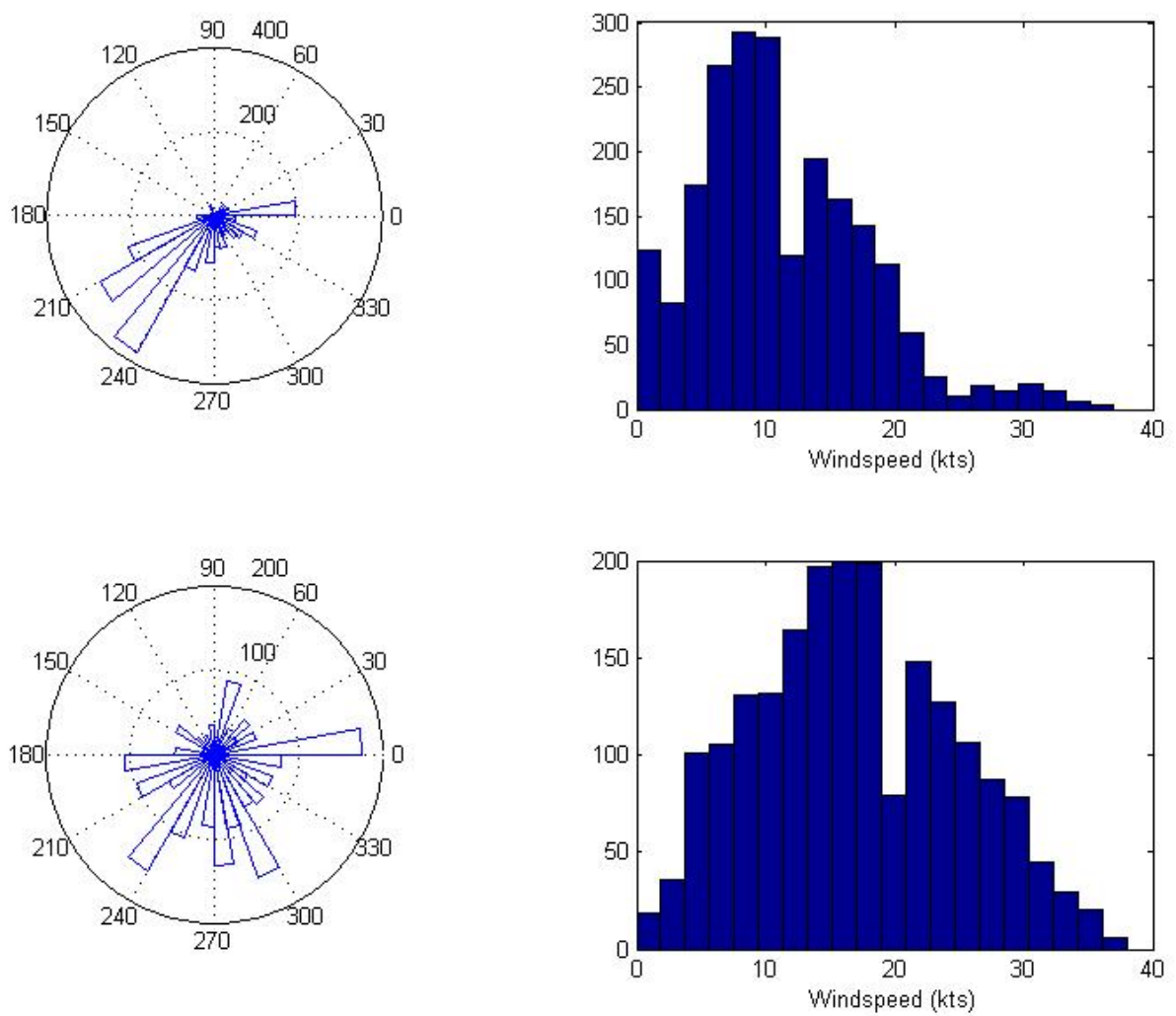

Figure 2: Charterhall (top) and Tiree (bottom) directional histograms (left) and windspeed histograms (right) for January days between 2003 and 2005.

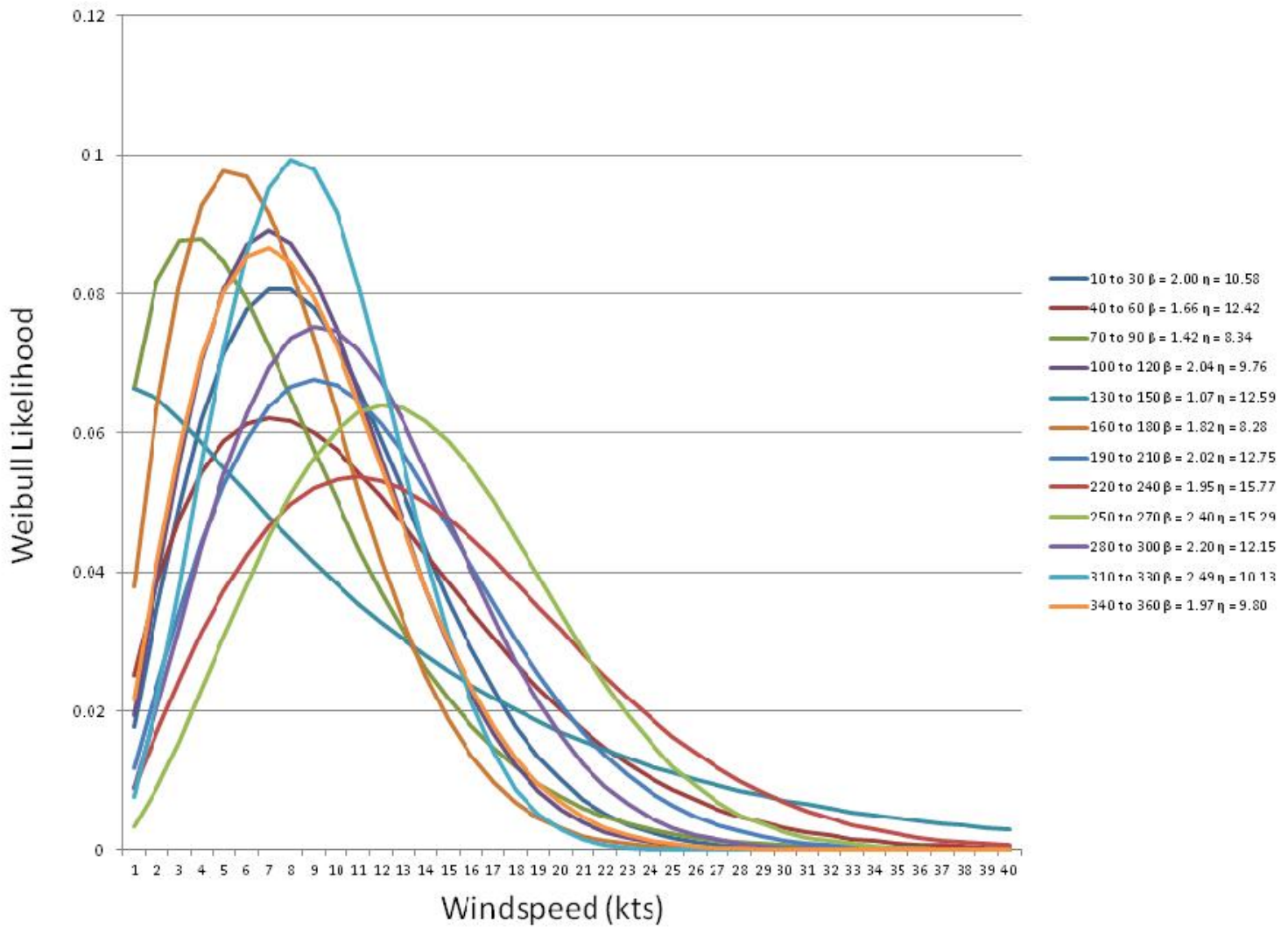

Figure 3: Weibull speed distributions fitted to binned directional components for Charterhall quarter 1 days in 2003 to 2005. 


\section{Cylindrical Mixture Distributions}

The relationship between windspeed and direction could be captured by extending the histogram into 2 dimensions with the relative frequency of occurrence obtained by 'stacking' data points on top of one another, which is essentially proportional to the joint probability of wind speed $v$ and direction $\omega$ :

$$
n_{v \omega} \propto P(v, \omega)
$$

A drawback of using histograms is the need to have bin widths specified in advance which can drastically alter their shape. The use of parametric distributions such as the Weibull provides a compact representation of event frequency which can be used for comparison and anomaly detection. There are 3 key design choices to be addressed in order to formulate a model which accommodates a range of complex wind speed and direction characteristics without requiring manual selection. First is the choice of joint distribution: it must allow the dependency between the two variables to be represented, it must accommodate the circular nature of the wind direction data and it must be able to accommodate the 2 critical features of wind speed - strictly positive and null wind. Second it must be able to accommodate multiple regimes at a site represented as multimodality. Lastly, the parameters of the distribution must be able to be inferred or estimated from an exemplar data set and in such a way that the process is largely automated.

\section{a. Circular Linear Distributions}

The second issue, as noted in [25], is that directional observations are periodic, that is to say they 'wrap around' and have no concept of extrema. To model a periodic variable $\omega$, the von Mises distribution is often used:

$$
P(\omega)=\frac{1}{2 \pi I_{0}(\kappa)} \exp \{\kappa \cos (\omega-\eta)\}
$$

This has two parameters $\eta$, which specifies the point on the circle where observations are most concentrated and $\mathrm{k}$, the dispersion of these points around $\eta$. $I_{0}$ is the zero order Bessel function. The von Mises distribution was employed by [22] in modelling wind direction implied by scatterometer data and in [18] to model wind direction but without any dependence structure assumed. The cylindrical data model of [23] proposed a joint distribution for bivariate data that modelled a linear and a periodic variable. Employing it for windspeed $v$ and wind direction $\omega$, would amount to a product of the respective probability density functions, giving

$$
P(\omega, v)=f(\omega) g(v \mid \omega)
$$

With $f$ taking the form of a Von Mises distribution:

$\omega \sim M(\eta, \kappa)$

And $g$ being a Gaussian distribution conditioned on the circular variable: 
$v \sim N\left(\begin{array}{l}\mu+\frac{\sigma}{\sqrt{\kappa}}\left[\rho_{1}(\cos \omega-\cos \eta)\right. \\ \left.-\rho_{2}(\sin \omega-\sin \eta)\right], \sigma^{2}\left(1-\rho^{2}\right)\end{array}\right)$

This assumes linear dependence of the conditional windspeed distribution on the direction which may be a limitation. Another shortcoming with this model is that in wind speed modelling there is the need to deal with 'calms' or 'null winds' when the wind speed is zero. An alternative candidate that is both computationally tractable and offers good performance in the surveys of [8] and [9] was the truncated Gaussian. Applying the non negativity constraint to the linear variable and entering the terms for wind speed and direction results in the following probability density function [24]:

$$
P(v)=\alpha_{0} \delta(v)+\left(1-\alpha_{0}\right) \frac{1}{\frac{1}{\sigma^{2}} \sqrt{2 \pi} \int_{0}^{\infty} \exp \left[\frac{-(v-\mu)^{2}}{2 \sigma^{2}}\right] d v} \exp \left[\frac{-(v-\mu)^{2}}{2 \sigma^{2}}\right]
$$

The parameter $\alpha$ is the frequency of the wind speed being zero at a given site and $\delta$ is the Dirac delta function. Substituting (2) and (6) into (3) gives the following expression for the joint distribution of wind speed and direction:

$$
P(v, \omega)=\alpha_{0} \delta(v)+\left(1-\alpha_{0}\right) \frac{\exp \left\{\kappa \cos (\omega-\eta)-\frac{(v-\mu(\omega))^{2}}{2 \sigma^{2}\left(1-\rho^{2}\right)}\right\}}{2 \pi^{\frac{3}{2}} I_{0}(\kappa) \sqrt{\sigma^{2}\left(1-\rho^{2}\right)} \int_{0}^{\infty} \exp \left[\frac{-(v-\mu)^{2}}{2 \sigma^{2}}\right] d v}
$$

where $\eta$ is the periodic mean, $\mu$ is a function of the linear and periodic means, $\sigma$ is the linear standard deviation, $\mathrm{k}$ is the dispersal of the periodic variable, $\rho$ is the correlation between the two variables.

\section{b. Finite Mixture Models}

A key difficulty of modelling any probability is the choice of distribution and additional variables compound this problem; although a number of distributions such as Weibull and Rayleigh are commonplace for wind speed representation, there are no multivariate equivalents. Even if such a distribution did exist, modelling the features of such a complex problem space are likely to be outwith the capabilities of a classical probability distribution, which are generally unimodal with a uniform dependency structure over their range. This limitation can be circumvented using a Finite Mixture Model [28] which allows an arbitrary shaped probability density to be approximated by a linear combination of simpler parametric densities. Formally, for a distribution with parameter set $\theta$, this can be expressed as

$$
P(v, \omega)=\sum_{i=1}^{M} P\left(\theta_{i}\right) P\left(v, \omega ; \theta_{i}\right)
$$

where $P\left(u, \omega ; \theta_{i}\right)$ is a parameterized distribution representing the $i^{\text {th }}$ mixture component and $P\left(\theta_{i}\right)$ is the corresponding mixing proportion which weights the contribution of a mixing component in the overall likelihood function. If the observation vector was defined as

$$
x=[v, \omega]^{T}
$$


Then, in a mixture of joint densities of the form given in (7), the $i^{\text {th }}$ component will have the following parameter vector:

$\theta_{i}=\left\{\mu_{i}, \eta_{i}, \sigma_{i}^{2}, \kappa_{i}, \rho_{i}\right\}$

Various model selection criteria can be applied to find the optimal number of mixture components for a given data set [18].

c. Parameter Estimation using the EM algorithm

A mixture model can be seen as what is known as an incomplete data problem, since the indication of which observations come from which mixture components is not present in the observed data set. For a set of observations $X$, a set of parameters $\theta$ for the $M$ distributions can be learned from data using some iterative approximation method such as Expectation Maximization [29] or Monte Carlo methods. The Expectation Maximization (EM) algorithm [29, 30] can be used to provide an iterative solution to incomplete data problems by synthesising missing data and thus turning them into more tractable complete data problems. The algorithm performs two steps on each of its iterations: on the Expectation (E) step, the expected value of the complete data is estimated while on the following Maximisation (M) step, the model parameter values that increase this expected value are obtained. EM has advantages over least squares and traditional gradient type approximations in that it does not require any assumptions regarding constraints or setting of step sizes [31], the choice of which may influence the algorithms stability. The following summarises the EM algorithm for a set of model parameters $\theta$ from a set of complete data X:

1. Set $\mathrm{i}=0$;

2. Set $\theta^{i}=\theta^{*}$

3. do

4. $Q\left(\theta ; \theta^{i}\right)=E_{\theta}\{\log P(\theta \mid X) \mid X\}$

5. Choose $\theta^{i}$ such that $Q\left(\theta^{i+1} ; \theta^{i}\right) \geq Q\left(\theta ; \theta^{i}\right)$

6. Set $\mathrm{i}=\mathrm{i}+1 ; \theta^{i}=\theta^{i+1}$

7. while $P\left(\theta^{i+1} \mid X\right)-P\left(\theta^{i} \mid X\right)$ is not arbitrarily small

In step 2 of the above, $\theta^{*}$ are the initial estimates of the model parameter values; these can be randomly assigned, chosen using heuristics or estimated using a simpler model. For an $M$ component mixture model presented with $\mathrm{N}$ observations, the Expected value of the likelihood is given by:

$Q=E\left[\sum_{j=1}^{N} \sum_{i=1}^{M} P\left(\theta_{i} \mid x_{j}\right) P\left(\theta_{i}\right) P\left(x_{j} \mid \theta_{i}\right)\right]$

Evaluating this requires the calculation of $P(\theta \mid x)$ from data, which through Bayes theorem can be obtained as:

$P\left(\theta_{i} \mid x_{j}\right)=\frac{P\left(\theta_{i}\right) P\left(x_{j} \mid \theta_{i}\right)}{P\left(x_{j}\right)}$ 


$$
P\left(\theta_{i} \mid x_{j}\right)=\frac{P\left(\theta_{i}\right) P\left(x_{j} \mid \theta_{i}\right)}{\sum_{m=1}^{M} P\left(\theta_{m}\right) P\left(x_{j} \mid \theta_{m}\right)}
$$

$Q$ is evaluated every iteration at the E-Step using the current model parameter values to effectively synthesize complete data by filling in the mixture contributions. Following this the parameter values are then updated on the M-Step. In the case of the distributions used here, these are tractable and can be found through Maximum Likelihood Estimation (MLE) i.e. by looking for saddle points in the likelihood function. As an example, the mixing proportions can be obtained by:

$$
P\left(\theta_{i}\right)=\frac{1}{N} \sum_{j=1}^{N} P\left(\theta_{i} \mid x_{j}\right)
$$

M-Step MLE update expressions for the mean and covariance terms for the linear variable and the circular mean and dispersal parameters are elaborated upon in [26, 29, 32].

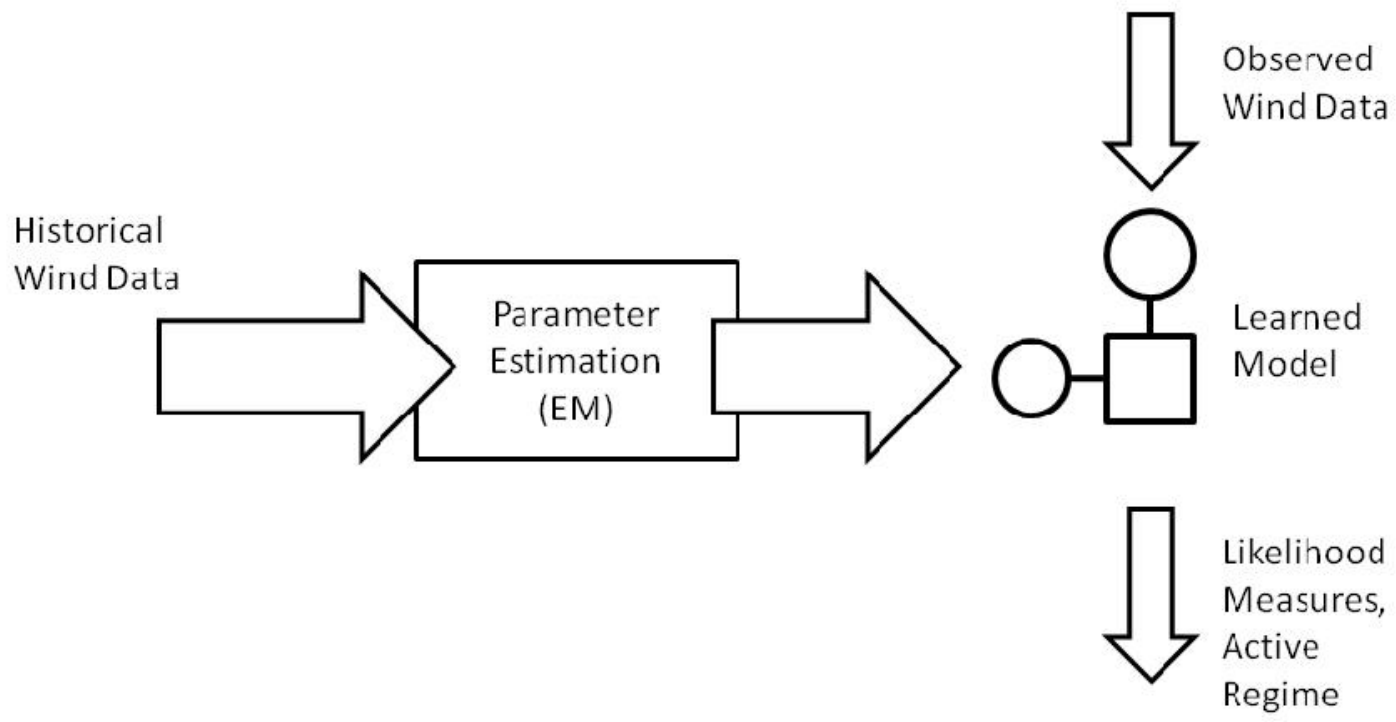

Fig 4: The process by which the model is generated from historical data and then applied to subsequent meteorological observations.

Figure 3 shows where the parameter estimation algorithm fits within the model application process - new observations do not necessitate retraining of the model.

\section{Station Model Selection}

Selecting model complexity is a key problem in modelling multimodal densities with mixture distributions; assuming too many components results in a rough distribution that generalizes poorly owing to its isolation of individual data points - phenomena known as 'overfitting'. While assuming too few modes results in a distribution that is overly general and obscures features of the data such as multiple modes or voids (places where data does not occur). In order to find the optimal number of components or modes, the complete data likelihood at the convergence of the learning phase can be used to find which model parameterization is the best fit to the data. In [29], a number of alternative model selection methods are discussed that go further by penalizing model complexity to preserve generalization capabilities. A limitation of maximum likelihood is that it prefers complex 
models, in the case of mixture distributions, adding more mixture components increases the likelihood until the situation is reached where a mixture has been mean centred on every data point. To avoid this, Bayesian Information Criterion (BIC) [33] was used. BIC is a model likelihood measure that includes a term that penalises more parameters being added to the model:

$B I C(X, \Lambda)=\sum_{n=1}^{N} \log P\left(x_{n} \mid \theta\right)-\frac{c}{2} \log N$

Choosing a model, $\Lambda$, with parameters $\vartheta$, based on BIC results in the most likely model given $N$ data points and the smallest parameter set size $c$.Table II shows the result of model order selection for all sites for the 1st quarter of the years 2003-2005. For each site, a model was trained with between 2 and 20 components on 1st quarter 2003-2005 data and the one with the lowest BIC selected.

\begin{tabular}{|c|c|c|c|}
\hline Station & Max LL & $\mathrm{BIC}$ & Optimal Components \\
\hline Charterhall & -28026 & -29671 & 8 \\
\hline Dunstaffnage & -30357 & -30722 & 5 \\
\hline Inverbervie & -32503 & -33202 & 8 \\
\hline Islay & -33046 & -33168 & 4 \\
\hline Kinloss & -29834 & -30468 & 18 \\
\hline Kirkwall & -30790 & -31204 & 12 \\
\hline Lerwick & -32871 & -33423 & 18 \\
\hline Lossiemouth & -31128 & -31646 & 14 \\
\hline Prestwick & -28839 & -29377 & 17 \\
\hline Skye & -27619 & -29373 & 21 \\
\hline Spade Adam & -26654 & -28646 & 17 \\
\hline Stornoway & -31711 & -31741 & 4 \\
\hline Tiree & -31337 & -31607 & 8 \\
\hline West Freugh & -29575 & -31383 & 3 \\
\hline Wick & -31317 & -31671 & 11 \\
\hline
\end{tabular}

Table II: Optimal Model Parameterization and Order Selection Criteria

Two features of the models learned from the sites are noteworthy: all require very different parameterisations again reinforcing the hypothesis that no one distribution is universally ideal and, given the high number of mixture components required to approximate the distribution of sites such as Skye, the complexity of the implied distributions goes beyond what a parameterised distribution could represent.

\section{Case Study: Charterhall}


Taking the case of Charterhall, an inland airfield on the east of Scotland, model order selection has shown the model for the first quarter of 2003-2005 for this site has 8 components, the parameters of which are listed in Table III. Figure 5 shows an annotated detail of the cylindrical Charterhall model, unrolled into a contour density plot for clarity.

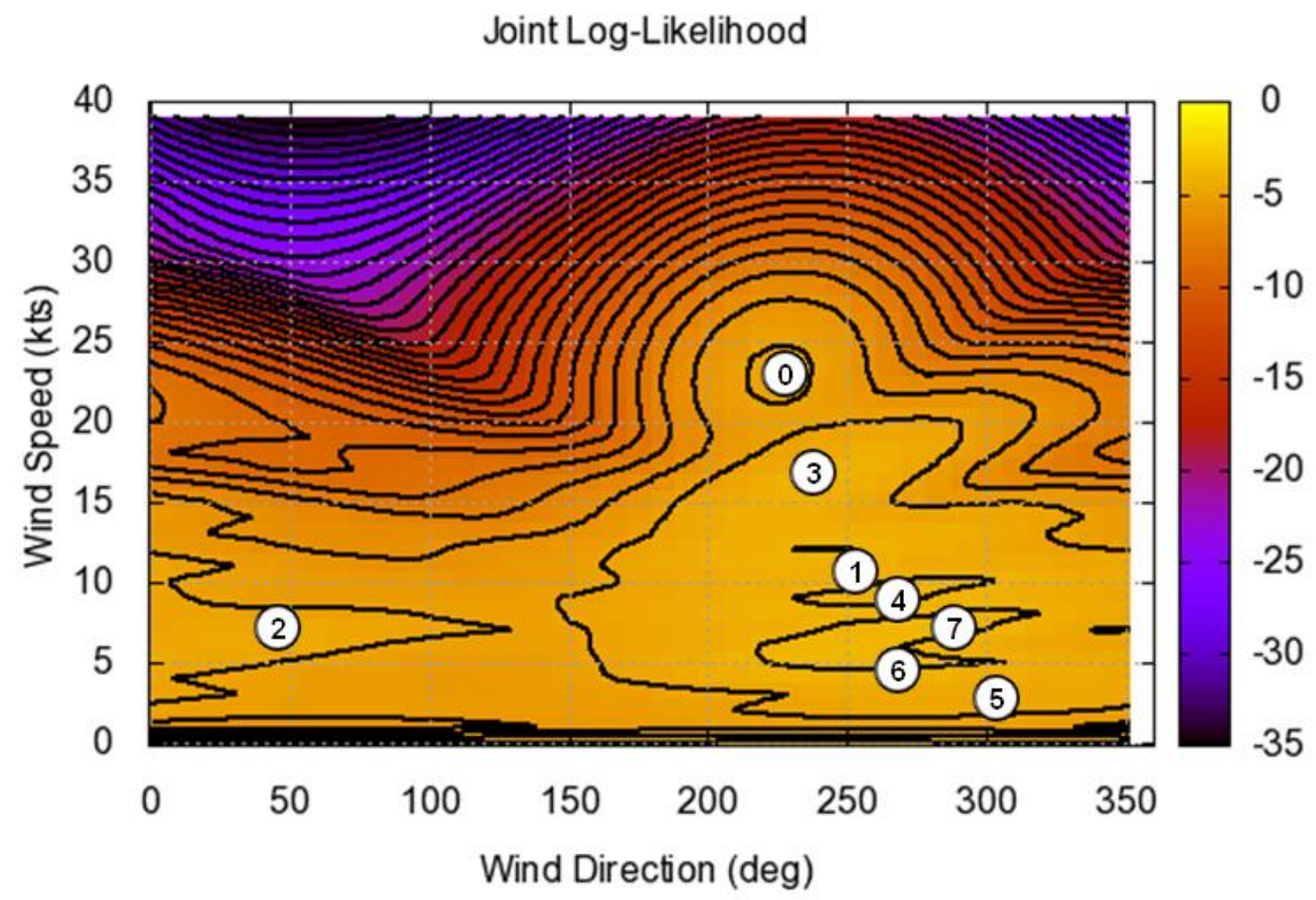

Figure 5: Unrolled contour density plot for Charterhall Q1 '03-'05 showing the multimodal and non-linear relationship between windspeed and direction. The positions of the means for each mixture component are marked.

\begin{tabular}{|c|c|c|c|c|c|c|c|}
\hline $\mathrm{i}$ & $\mathrm{P}\left(\theta_{\mathrm{i}}\right)$ & $\mathrm{\eta}\left(^{\circ}\right)$ & $\mu(\mathrm{kts})$ & $\mathrm{K}$ & $\sigma^{2}$ & $\rho_{1}$ & $\rho_{2}$ \\
\hline & & & & & & & \\
\hline 0 & 0.06 & 226 & 23 & 8.31 & 9.3 & 0.10 & -0.07 \\
\hline 1 & 0.15 & 251 & 12 & 1.1 & 3.7 & 0.8 & -0.004 \\
\hline 2 & 0.06 & 48 & 6 & 1.62 & 1.06 & 0.08 & 0.44 \\
\hline 3 & 0.15 & 239 & 16 & 2.49 & 8.28 & 0.76 & -0.13 \\
\hline 4 & 0.19 & 268 & 9 & 0.77 & 3.3 & 0.84 & 0.1 \\
\hline 5 & 0.12 & 302 & 2.5 & 0.38 & 1.8 & 0.55 & 0.23 \\
\hline 6 & 0.18 & 266 & 5 & 0.9 & 0.65 & 0.5 & 0.04 \\
\hline 7 & 0.14 & 271 & 7 & 1.17 & 2.0 & 0.82 & 0.24 \\
\hline
\end{tabular}

The light areas in the figure 4 indicate regions of increasing observation likelihood with numbered white circles indicating the position of the mixture component means. Wind speed and direction 
observations closest, in terms of maximised likelihood, to these mixture components can be seen as being part of a particular behaviour or regime that occurs commonly at the site associated with the model. From table III, wind regimes captured by components 4 and 6 have an almost equal likelihood of occurrence as their mixing proportions $\left(P\left(\theta_{i}\right)\right)$ imply. Both regimes are westerly but the one associated with component 4 has a higher wind speed with greater variability. Component 0 features a high wind speed with high variance but its mixing proportion indicates a low probability of occurring. The regime captured by component 3 has a windspeed of $16 \mathrm{kts}$ and a dependence on direction - speed increases as the direction rotates from westerly towards north westerly. Component 2, representing lower speed north easterly winds, is a less common occurring regime. Also notable from figure 5 are the voids in the probability density - the regions of low likelihood (dark areas in the graph) show that strong south-easterly winds are highly unlikely.

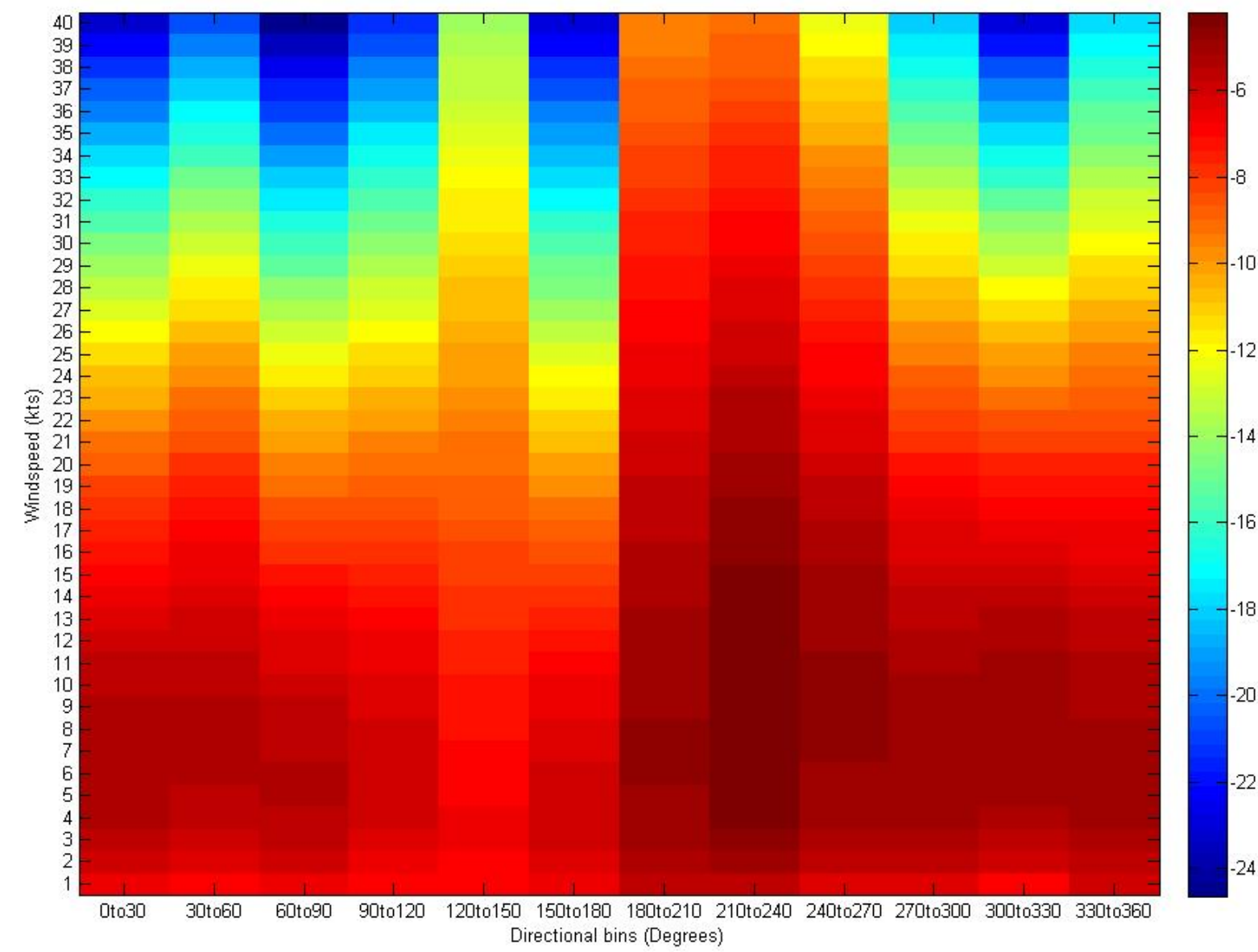

Figure 6: 2 Dimensional histogram representation of a joint distribution of wind direction and speed formed from the histogram implied by a 12 sector wind rose and two parameter Weibull distributions at Charterhall Q1 '03-'05.

Returning to the more conventional methods for wind speed and direction demonstrated in figure 3, figure 6 shows how Charterhall 1st quarter 2003-2005 wind observations are captured with the ensemble of 12 direction segments, 2-parameter Weibull distributions. The main mode is captured in the westerly direction along with a second, less likely, directional mode, at lower speed in the north easterly direction. Although a far coarser representation (higher wind speeds are deemed likely in the westerly direction by the 2-parameter Weibull distributions), this still highlights the differences in behaviour in different directions.

An additional benefit of using the mixture representation is that the regimes identified through model training can be captured as they recur by choosing the most likely mixture component for a given speed and direction observation pair; Figure 7 shows the co-incident wind speed and direction observations against time for Charterhall in Q1 2006. Along side these are the mixture component 
label representing the active regime implied by the observation pair as well as the associated observation likelihood. Some regimes are fairly stable such as the one represented by component 5 and a site can remain in one for several days at a time as the series around January 7th shows.

\section{Resource Assessment}

A longer term goal of this research is to produce realistic simulation of wind farm generation output. Incorporating the temporal aspects into a future formulation would yield a truly generative model, that is, one whose parameterization incorporates all information about the stochastic properties of the process, random samples can be drawn from the probability distribution to simulate that modelled process. Coupling this with models of plant performance and efficiency $[34,35]$ could in

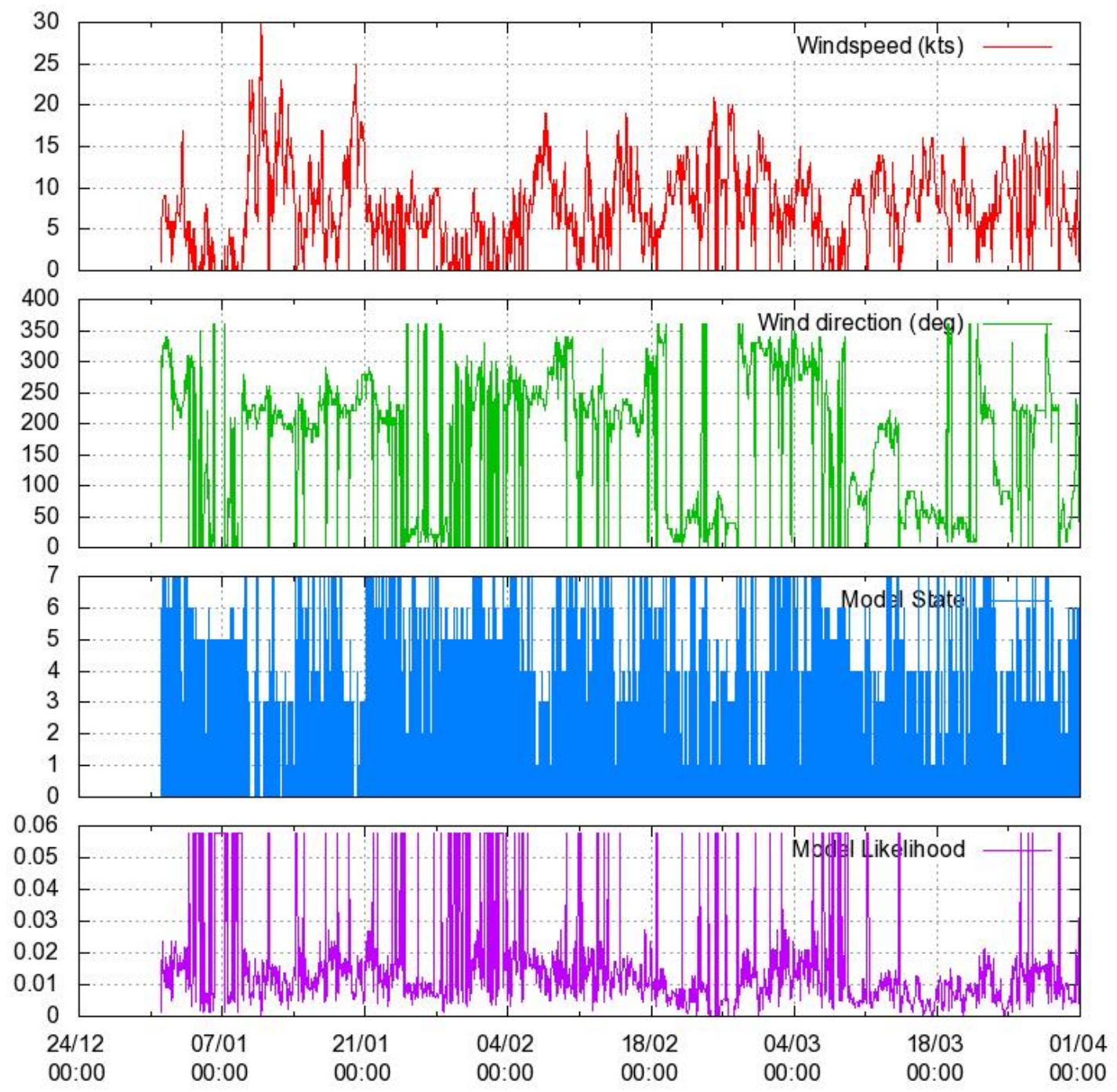

Figure 7: Model output; from top, wind speed then direction time series, ddominant mixture components/regime (2nd from bottom) and model likelihoods (bottom) for Charterhall in the first quarter of 2006.

turn be used to simulate future generation scenarios. In order to draw samples from the Cylindrical Mixture, multinomial samples across the mixing proportions are drawn [36] followed by samples from the Von Mises marginal [37] which are then used to draw from the conditional Cylindrical distribution. Slice Sampling [38] is used to draw samples from the conditional Cylindrical distribution along with the directional samples already obtained. Table IV shows the potential value of the proposed approach over existing methods. Weibull distributions of increasing directional resolution 
(1, 4 and 12 directional sectors) were fitted using least squares for the case of Charterhall, and Monte Carlo samples drawn for 8760 hours.

\begin{tabular}{|c|c|c|c|}
\hline Model & Yield @ 2MW MWh & Yield error @ 2MW MWh & $f|\varepsilon|$ \\
\hline & & & \\
\hline Raw data & 3968.1 & n/a & n/a \\
\hline Weibull 1-sector & 4360.6 & 392.5 & 29,828 \\
\hline Weibull 4-sector & 4357.6 & 389.5 & 29,605 \\
\hline Weibull 12-sector & 4300.0 & 331.9 & 25,226 \\
\hline Cylinder Mixture & 3862.0 & -106.1 & 8060 \\
\hline
\end{tabular}

Table IV: Charterhall Q1 Model Comparison

The wind speed estimates were converted to power output via application of a per-unit power curve [39]. Hub height conversion was achieved via log power law, assuming a hub height of $80 \mathrm{~m}$, measurement height of $10 \mathrm{~m}$, and $z 0$ of 0.02 [40]. Assuming a single $2 \mathrm{MW}$ wind turbine, and electricity production credit of $f 76 / \mathrm{MWh}$, Table IV illustrates the increased accuracy gained by including directional information. This is expressed both in energy yield and economic accuracy. It can be seen that the energy yield estimate for the cylindrical model provides a more accurate estimate. It under-estimates the yield rather than the Weibull models, which consistently overestimate the yield by assuming the wind speed distribution is the same for all directions.

\section{Conclusions and Further Work}

This paper has presented a model that captures multimodal speed and direction characteristics, along with the dependency structure that relates them, of the wind resource at a given location. This uses existing meteorological instrumentation to provide a representation that breaks down the overall site behaviour into a combination of recurring sub-behaviours or regimes that are characterised by their direction and wind strength as well as the variability and relation between the two measurements. This has an advantage over the Weibull representation of windspeed as the mixture model approach adopted allows multimodal windspeed distributions to be captured as well as associating the modes with a particular direction and level of variability - high directional variability may compromise the level of generation capable at a given site. Building models of wind resource that accommodate more complex wind dynamics could be employed with the addition of time series parameterization as a component in future active control systems [41]: a state estimator of wind resource allows a control system to execute appropriate blade pitching and rotor yawing in the presence of uncertain measurements which the cylindrical mixture provides through its component indicator label. Estimating directional variability allows loads on the turbine incurred by excessive yawing to be anticipated where Linear Gaussian type control models would not have represented expected direction and its associated variability adequately, The explicit wind directions may not be useful from the perspective of inter-site comparison where only the speed and consistency of direction are of interest, but for an intra-site comparison the homogeneity of the wind resource at various points in the site may govern or rule out the placement of turbines. In [42], a means of quantifying similarity of mixture models was demonstrated which could be readily formulated for the cylindrical distribution, providing a means of quantitatively comparing sites, which in the practical application will enable a planner/developer to find sites that behave in a similar way to optimally placed ones, as an alternative to spatial extrapolations [43], and in essence optimize the site design. 


\section{References}

[1] OfGEM. Renewables Obligation: Annual Report 2008-2009, 2010. Available at http://www.ofgem.gov.uk

[2] Pudjianto, D., Ramsay, C. \& Strbac, G. Virtual power plant and system integration of distributed energy resources. Renewable Power Generation, IET, vol.1, no.1, pp.10-16, March 2007

[3] Dielmann, K. \& van der Velden, A. Virtual power plants (VPP) - a new perspective for energy generation? Modern Techniques and Technologies, 2003. MTT 2003. Proceedings of the 9th International Scientific and Practical Conference of Students, Post-graduates and Young Scientists, vol., no., pp. 18- 20, 7-11 April 2003

[4] Geidl, M. \& Andersson, G. A modeling and optimization approach for multiple energy carrier power flow. Power Tech, 2005 IEEE Russia, vol., no., pp.1-7, 27-30 June 2005

[5] Pinson, P. \& Madsen H. Adaptive modeling and forecasting of wind power fluctuations with Markov-switching autoregressive models. Journal of Forecasting 31(4), pp. 281-313, 2012.

[6] Tavner, P., Edwards, C., Brinkman, A. \& Spinato, F. Influence of Wind Speed on Wind Turbine Reliability, Wind Engineering vol. 30 iss. 1, pp. 55-72, Jan 2006.

[7] Wilson, G. \& McMillan, D. Modelling the effects of the environment on wind turbine failure modes using neural networks, IET International Conference on Sustainable Power Generation and Supply, Sep-2012

[8] Carta, J. A., Ramírez, P. \& Velázquez, S. A review of wind speed probability distributions used in wind energy analysis Case studies in the Canary Islands. Renewable and Sustainable Energy Reviews 13, pp. 933-955, 2009.

[9] Morgan, U.C., Lackner, M., Vogel, R.M. \& Baise, L.G. Probability distributions for offshore wind speeds. Energy Conversion and Management 52, pp. 15-26, 2011.

[10] Jaramillo, O. A. \& Borja, M. A. Wind speed analysis in La Ventosa, Mexico: a bimodal probability distribution case, Renewable Energy Volume 29, Issue 10, Pages 1613-1630, August 2004.

[11] Carta, J.A. \& Ramírez, P. Use of finite mixture distribution models in the analysis of wind energy in the Canarian Archipelago. Energy Conversion and Management 48, pp. 281-291, 2007.

[12] Pashardes, S. \& Christofides, C. Statistical analysis of wind speed and direction in Cyprus. Solar Energy, Volume 55, Issue 5, Pages 405-414, November 1995.

[13] Cancino-Solórzano, Y. \& Xiberta-Bernat, J. Statistical analysis of wind power in the region of Veracruz (Mexico). Renewable Energy Volume 34, Issue 6, Pages 1628-1634, June 2009.

[14] Sadhuram, Y. Seasonal variation of $\Sigma \theta$ with wind speed, direction and stability,BoundaryLayer Meteorology Volume: 34, Issue: 1, pp. 99-101, Springer Netherlands, 1986. 
[15] Antoniou, I., Wagner, R., Pedersen, S.M., Paulsen, U., Madsen, H.A., Jørgensen, H.E., Thomsen, K., Enevoldsen, P. \& Thesbjerg, L. "Influence of wind characteristics on turbine performance", Proc. European Wind Energy Conference and Exhibition, Milan, 7th-10th May 2007.

[16] Montes, E. , Arnedo, A. , Cordón, R. \& Zubiaur, R. Influence of wind shear and seasonality on the power curve and annual energy production of wind turbines. Power, No. 1, 2009.

[17] Sathe, A. \& Bierbooms, W. Influence of different wind profiles due to varying atmospheric stability on the fatigue life of wind turbines, J. Phys.: Conf. Ser. Vol. 75, 2007.

[18] IEC 61400-12 Wind turbines - Part 12-1: Power performance measurements of electricity producing wind turbines, IEC 2005-12-16.

[19] Llombart, A., Watson, S.J., Llombart, D. \& Fandos, J.M. 'Power Curve Characterization I: Improving the Bin Method' ICREPQ'05, Zaragoza 16-18 of March, 2005

[20] Carta, J.A., Bueno, C. \& Ramírez, P. Statistical modelling of directional wind speeds using mixtures of von Mises distributions: A case study. Energy Conversion \& Management, Vol. 49, Issue 5, pp. 897-907, May 2008.

[21] Qin, X., Zhang, J. \& Yan, X. A New Circular Distribution and Its Application to Wind Data, Journal of Mathematics Research, Vol. 2, No. 3, pp. 12-17; Canadian Center of Science and Education, August 2010.

[22] Carta, J.A., Ramírez, P. \& Bueno, C. A joint probability density function of wind speed and direction for wind energy analysis. Energy Conversion and Management 49, pp. 1309-1320, 2008.

[23] Akdag, S.A., Bagiorgas, H.S. \& Mihalakakou, G. Use of two-component Weibull mixtures in the analysis of wind speed in the Eastern Mediterranean. Applied Energy 87, pp. 2566-2573, 2010.

[24] Akpinar, S. \& Akpinar, E.K. Estimation of wind energy potential using finite mixture distribution models. Energy Conversion and Management 50, pp. 877-884, 2009.

[25] Cornford, D., Nabney, I.T. \& Bishop, C.M. "Neural network-based wind vector retrieval from satellite scatterometer data," in Neural Computing and Applications 8, pp. 206-217. 1999.

[26] Mardia, K.V. \& Sutton, T.W. "A Model for Cylindrical Variables with Applications," Journal of the Royal Statistical Society. Series B (Methodological), Vol. 40, No. 2, pp. 229-233, 1978.

[27] Takle, E.S. \& Brown, J. Note on the use of Weibull to characterize wind speed data. Journal of Applied Meteorology 17, pp. 556-559, 1978.

[28] McLachlan, G.J. \& Peel, D. Finite Mixture Models, New York: Wiley-Interscience, 2000.

[29] Dempster, A., Laird, N. \& Rubin, D. Maximum Likelihood from Incomplete Data via the EM Algorithm. Journal of the Royal Statistical Society, Series B. 1977.

[30] McLachlan, G.J. \& Krishnan, T. The EM Algorithm and Extensions, New York: WileyInterscience, 1997. 
[31] Maher, E.F. \& Laird, N.M. EM Algorithm Reconstruction of Particle Size Distributions from Diffusion Battery Data. Journal of Aerosol Science, Vol. 16, No. 6, pp. 557-570, Pergamon Press, 1985.

[32] Anderson-Cook, C.M. An extension to modeling cylindrical variables. Statistics and Probability Letters 35, pp. 215-223, Elsevier 1997.

[33] Kass, R.E. \& Raftery, A.E. Bayes Factors. Journal of the American Statistical Association Vol. 90, No. 430, pp. 773-795, American Statistical Association, June 1995.

[34] Kusiak, A., Zheng, H.-Y. \& Song, Z. Wind Farm Power Prediction: A Data-Mining Approach, Wind Energy Vol. 12, pp. 275-293, Wiley 2009.

[35] Stephen, B., Galloway, S. J., McMillan, D., Hill, D. C. and Infield, D. G. "A Copula Model of Wind Turbine Performance", IEEE Transactions on Power Systems Vol. 26, No. 2, pp. 965 - 966, 2011.

[36] Pederson, D.G. Note: An Approximate Method of Sampling a Multinomial Population, Biometrics , Vol. 29, No. 4 (Dec., 1973), pp. 814-821

[37] Best, D.J. \& Fisher, N.I. Efficient Simulation of the von Mises Distribution, Journal of the Royal Statistical Society. Series C (Applied Statistics), Vol. 28, No. 2 (1979), pp. 152-157

[38] Neal, R. M. "Slice sampling" (with discussion), Annals of Statistics, vol. 31, pp. 705-767, 2003.

[39] Garrad Hassan document 11914/BT/02. Tradewind project, WP2.6 -Equivalent Wind Power Curves. Available at: http://www.tradewind.eu/fileadmin/documents/publications/D2.4_Equivalent_Wind_Power_Curves_11914bt02c.pdf

[40] Wiernga, J. Representative roughness parameters for homogeneous terrain, in BoundaryLayer Meteorology, Vol. 63, No. 4, pp. 323-363, Springer Netherlands, 1993

[41] Kusiak, A., Song, Z. \& Zheng, H.-Y. Anticipatory Control of Wind Turbines with Data-Driven Predictive Models, IEEE Transactions on Energy Conversion, Vol. 24, No. 3, pp. 766-774, 2009

[42] Cadez, I.A., McLaren, C.E., Smyth, P. \& McLachlan, G.J. "Hierarchical Models for Screening of Iron Deficiency Anaemia," presented at the International Conference of Machine Learning, Santa Cruz, 1999.M.

[43] Gibescu, B., Ummels, C. \& Kling, W.L. Statistical Wind Speed Interpolation for Simulating Aggregated Wind Energy Production under System Studies, 9th International Conference on Probabilistic Methods Applied to Power Systems KTH, Stockholm, Sweden - June 11-15, 2006 
